\title{
Methodology for Determine the Moment of Disconnection of Patients of the Mechanical Ventilation using Discrete Wavelet Transform
}

\author{
H. González, H. Acevedo, C. Arizmendi \\ Control \& Mecatrónica Research Group \\ Universidad Autónoma de Bucaramanga
}

Avenida 42 No 48-11, Bucaramanga, Colombia

\{hgonzalez7, hacevedo3, carizmendi\}@unab.edu.co

\begin{abstract}
The process of weaning from mechanical ventilation is one of the challenges in intensive care units. 66 patients under extubation process (T-tube test) were studied: 33 patients with successful trials and 33 patients who failed to maintain spontaneous breathing and were reconnected. Each patient was characterized using 7 time series from respiratory signals, and for each serie was evaluated the discrete wavelet transform. It trains a neural network for discriminating between patients from the two groups.
\end{abstract}

Index Terms - Mechanical Ventilation, Time series from respiratory signals, Wavelet Transform, Neural Networks.

\section{INTRODUCTION}

Mechanical ventilators, which are often also called respirators, are used to artificially ventilate the lungs of patients who are unable to naturally breathe from the atmosphere. There are two main divisions of mechanical ventilation: invasive ventilation and non-invasive ventilation. There are two main modes of mechanical ventilation within the two divisions: positive pressure ventilation, where air (or another gas mix) is pushed into the trachea, and negative pressure ventilation, where air is essentially sucked into the lungs [1].

Discontinuation of mechanical ventilation, also called weaning or extubation, should be performed as soon as autonomous respiration can be sustained. It is one of the most challenging problems in intensive care units. Despite advances in mechanical ventilation and respiratory support, the science of determining if the patient is ready for extubation is still very imprecise. A failed weaning trial is discomforting for the patient and may induce significant cardiopulmonary distress. When mechanical ventilation is discontinued, up to 25 percent of patients have respiratory distress severe enough to necessitate reinstitution of ventilatory support. Hence the need for a more accurate prediction of the optimal disconnection time, which is extended to the whole weaning process [2-3]. The variability of breathing pattern is not random and can be explained by central neural mechanisms or instability of the

\author{
B.F. Giraldo, Senior Member IEEE
}

\author{
Dept.of ESAII, Universitat Politècnica de Catalunya, \\ Institut de Bioenginyeria de Catalunya (IBEC) and \\ CIBER de Bioingeniería, Biomateriales y Nanomedicina \\ (CIBER-BBN). \\ c.. Pau Gargallo, 5, 08028, Barcelona, Spain \\ beatriz.giraldo@upc.edu
}

feedback loops [4]. This variability was analyzed previously in $[5-8]$.

The purpose of this work is to characterize the variability of the respiratory pattern of patients on weaning trials using neural network techniques, providing enhanced information in order to identify patients with successful spontaneous breathing trials and patients with unsuccessful trials. Neural networks are sophisticated statistical techniques capable of modeling extremely complex functions. Wavelet transform was used as the kernel of transformation, to obtain the statistics average, standard deviation, kurtosis, interquartile range from the decomposition coefficients, instead of the raw data; these are the training data for the neural network. Some authors have proposed methodologies to give solution to this problem using neural networks and support vector machine [9$11]$.

\section{ANALYZED DATA}

In this study, respiratory flow signals were measured in 66 patients under mechanical ventilation and extubation process (database WEANDB). The patients were recorded in the Departments of Intensive Care Units at Santa Creu i Sant Pau Hospital, Barcelona, Spain,and Getafe Hospital, Getafe, Spain, according to the protocols approved by the local ethics committees.

The patients were submitted under T-tube test, disconnected from the ventilator and maintained spontaneous breathing through an endotraqueal tube during $30 \mathrm{~min}$. According to the clinical criteria, the patients were classified into two groups: group A, 33 patients whose T-tube test was overcome successfully, and group B, 33 patients who failed the test and therefore could not be extubated.

The respiratory flow was obtained with a pneumotachograph (Datex-Ohmeda monitor with variable reluctance transducer) connected to an endotracheal tube. The signals were recorded at a sampling frequency of $250 \mathrm{~Hz}$ during 30 minutes.

The respiratory pattern can be characterized by the following time series: inspiratory time $\left(\mathrm{T}_{\mathrm{I}}\right)$, expiratory time 
( $\mathrm{TE}_{\mathrm{E}}$, breathing cycle duration ( $\mathrm{T}_{\mathrm{Tot}}$ ), tidal volume (VT), inspiratory fraction ( $\left.\mathrm{T}_{\mathrm{I}} / \mathrm{T}_{\mathrm{Tot}}\right)$, mean inspiratory flow $\left(\mathrm{V}_{\mathrm{T}} / \mathrm{T}_{\mathrm{I}}\right)$ and rapid shallow breathing (f/VT), were $\mathrm{f}$ is respiratory rate. The figure 1 shows a respiratory signal and the respective parameters.
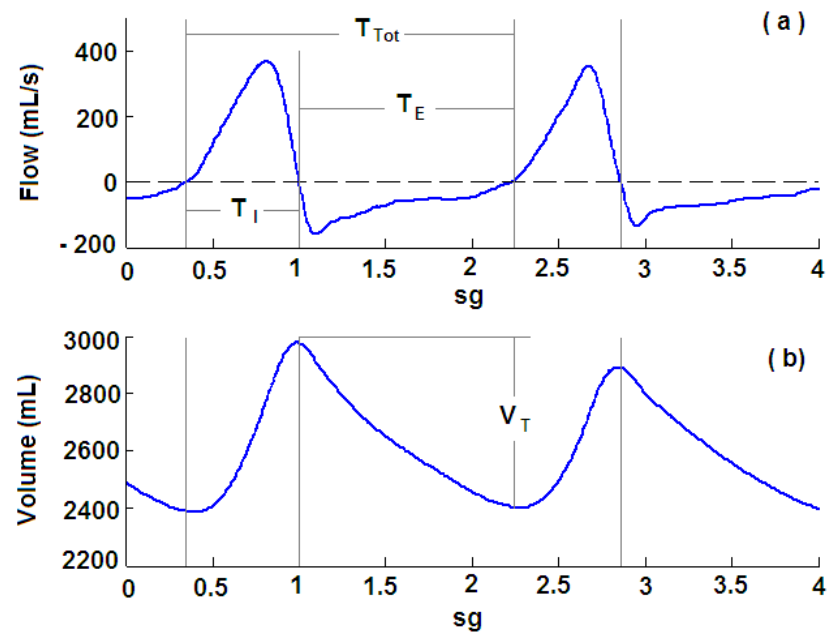

Figure 1. (a) Respiratory flow signal and their time series: inspiratory time (TI), expiratory time (TE) and breathing cycle duration (TTot). (b) Respiratory volume signal and tidal volume (VT).

\section{WAVELET TRANSFORM}

The Continuous Wavelet Transform (CWT) of a signal $x(t)$ with mother wavelet $\psi(\cdot)$ is defined as:

$$
W(\tau, s)=\frac{1}{\sqrt{|s|}} \int_{-\infty}^{\infty} x(t) \psi\left(\frac{t-\tau}{s}\right) d t
$$

being the transformed signal $W(\tau)$ is a function of the translation parameter $\tau$ and the scale $s$. The signal energy is normalized dividing the wavelet coefficients by $1 / \sqrt{|s|}$. The original signal can be reconstructed with the inverse CWT, defined by:

$$
\begin{aligned}
x(t) & =\frac{1}{C_{\psi}^{2}} \int_{-\infty}^{\infty} \int_{-\infty}^{\infty} W(\tau, s) \frac{1}{s^{2}} \psi\left(\frac{t-\tau}{s}\right) d \tau d s \\
C_{\psi} & =\int_{-\infty}^{\infty} \frac{|\psi(f)|^{2}}{f} d f<\infty
\end{aligned}
$$

However many real applications need to be defined in a discrete domain. An important development for the application of wavelet theory in discrete signal processing was presented in [12], using Multirresolution Analysis. The Discrete Wavelet Transform (DWT) is implemented via an octave filter bank, as a cascade of low-pass L(z) and high-pass $\mathrm{H}(\mathrm{z})$ filters, followed by subsampling. Every pair of filters represents a decomposition level. The reconstruction of the original signal is possible using the synthesis filter bank where the signals are upsampled and passed through the filters L'( $\mathrm{z})$ and $H^{\prime}(z)$. The reconstruction procedure leads to the restoration of the original signal, if the coefficient is not altered.

Selection of the mother wavelet orders. The DWT was

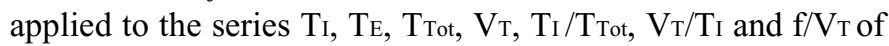
the 66 patients. Three mother wavelets were used to implement the DWT: Daubechies, Coiflet and Symlet. For every mother wavelet the signal was decomposed in three levels, later the signal was reconstructed and it was estimated the mean square error (MSE) and signal-to-noise ratio (SNR). In Eq. 3 and $4, x$ is the original signal, and $\hat{x}$ is the reconstructed signal.

$$
\begin{aligned}
& M S E=\frac{1}{n} \sum_{i=1}^{n}(x(i)-\hat{x}(i))^{2} \\
& S N R=10 \log \left[\sum_{i=1}^{n} \frac{(x(i))^{2}}{M S E * n}\right]
\end{aligned}
$$

In the Table I and II are reported the average value of MSE and SNR indices obtained for the patients of both groups, A and $\mathrm{B}$. The Daubechies function yields smaller MSE.

TABLE I

MSE INDEX

\begin{tabular}{|c|c|c|c|c|}
\hline Signal & Group & Daubechies & Coiflets & Symlets \\
\hline \multirow{2}{*}{$\mathrm{T}_{\mathrm{I}}$} & $\mathrm{A}$ & $1.2977 \mathrm{e}-1$ & $4.0353 \mathrm{e}-1$ & $3.5163 \mathrm{e}-1$ \\
\cline { 2 - 5 } & $\mathrm{B}$ & $4.9177 \mathrm{e}-2$ & $1.7426 \mathrm{e}-1$ & $1.6509 \mathrm{e}-1$ \\
\hline \multirow{2}{*}{$\mathrm{TE}_{\mathrm{E}}$} & $\mathrm{A}$ & $1.1760 \mathrm{e}-1$ & $4.5919 \mathrm{e}-1$ & $3.8479 \mathrm{e}-1$ \\
\cline { 2 - 5 } & $\mathrm{B}$ & $1.5225 \mathrm{e}-1$ & $5.8055 \mathrm{e}-1$ & $4.4476 \mathrm{e}-1$ \\
\hline \multirow{2}{*}{$\mathrm{T}_{\mathrm{T}} \mathrm{t}$} & $\mathrm{A}$ & $2.3809 \mathrm{e}-1$ & $9.3449 \mathrm{e}-1$ & $7.7503 \mathrm{e}-1$ \\
\cline { 2 - 5 } & $\mathrm{B}$ & $2.0703 \mathrm{e}-1$ & $8.1778 \mathrm{e}-1$ & $6.4462 \mathrm{e}-1$ \\
\hline \multirow{2}{*}{$\mathrm{V}_{\mathrm{T}}$} & $\mathrm{A}$ & $8.7888 \mathrm{e}+3$ & $4.1917 \mathrm{e}+4$ & $3.2159 \mathrm{e}+4$ \\
\cline { 2 - 5 } & $\mathrm{B}$ & $9.8233 \mathrm{e}+3$ & $5.7056 \mathrm{e}+4$ & $4.1572 \mathrm{e}+4$ \\
\hline \multirow{2}{*}{$\mathrm{T}_{\mathrm{I}} / \mathrm{T}_{\mathrm{Tot}}$} & $\mathrm{A}$ & $2.2343 \mathrm{e}-3$ & $7.0119 \mathrm{e}-3$ & $6.4097 \mathrm{e}-3$ \\
\cline { 2 - 5 } & $\mathrm{B}$ & $1.4937 \mathrm{e}-3$ & $5.3575 \mathrm{e}-3$ & $4.7362 \mathrm{e}-3$ \\
\hline \multirow{2}{*}{$\mathrm{V}_{\mathrm{T}} / \mathrm{T}_{\mathrm{I}}$} & $\mathrm{A}$ & $8.0140 \mathrm{e}+3$ & $4.0733 \mathrm{e}+4$ & $3.1110 \mathrm{e}+4$ \\
\cline { 2 - 5 } & $\mathrm{B}$ & $1.4724 \mathrm{e}+4$ & $8.9672 \mathrm{e}+4$ & $6.2271 \mathrm{e}+4$ \\
\hline \multirow{2}{*}{$\mathrm{f} / \mathrm{V}_{\mathrm{T}}$} & $\mathrm{A}$ & $1.2117 \mathrm{e}-3$ & $4.8754 \mathrm{e}-3$ & $4.3667 \mathrm{e}-3$ \\
\cline { 2 - 5 } & $\mathrm{B}$ & $1.6005 \mathrm{e}-3$ & $9.6664 \mathrm{e}-3$ & $7.1020 \mathrm{e}-3$ \\
\hline
\end{tabular}

TABLE II

SNR INDEX

\begin{tabular}{|c|c|c|c|c|}
\hline Signal & Group & Daubechies & Coiflets & Symlets \\
\hline \multirow{2}{*}{$\mathrm{T}_{\mathrm{I}}$} & $\mathrm{A}$ & $1.7260 \mathrm{e}+1$ & $1.1530 \mathrm{e}+1$ & $1.2114 \mathrm{e}+1$ \\
\cline { 2 - 5 } & $\mathrm{B}$ & $1.7687 \mathrm{e}+1$ & $1.1881 \mathrm{e}+1$ & $1.2489 \mathrm{e}+1$ \\
\hline \multirow{2}{*}{$\mathrm{TE}_{\mathrm{E}}$} & $\mathrm{A}$ & $1.6700 \mathrm{e}+1$ & $1.0564 \mathrm{e}+1$ & $1.1367 \mathrm{e}+1$ \\
\cline { 2 - 5 } & $\mathrm{B}$ & $1.5974 \mathrm{e}+1$ & $9.2988 \mathrm{e}+0$ & $1.0400 \mathrm{e}+1$ \\
\hline \multirow{2}{*}{$\mathrm{T}_{\mathrm{T}}+\mathrm{A}$} & $\mathrm{A}$ & $1.8261 \mathrm{e}+1$ & $1.1836 \mathrm{e}+1$ & $1.2770 \mathrm{e}+1$ \\
\cline { 2 - 5 } & $\mathrm{B}$ & $1.7764 \mathrm{e}+1$ & $1.1028 \mathrm{e}+1$ & $1.2253 \mathrm{e}+1$ \\
\hline \multirow{2}{*}{$\mathrm{V}_{\mathrm{T}}$} & $\mathrm{A}$ & $1.6621 \mathrm{e}+1$ & $9.4782 \mathrm{e}+0$ & $1.0782 \mathrm{e}+1$ \\
\cline { 2 - 5 } & $\mathrm{B}$ & $1.7779 \mathrm{e}+1$ & $1.0098 \mathrm{e}+1$ & $1.1473 \mathrm{e}+01$ \\
\hline \multirow{2}{*}{$\mathrm{T}_{\mathrm{I}} / \mathrm{T}_{\mathrm{Tot}}$} & $\mathrm{A}$ & $2.0430 \mathrm{e}+1$ & $1.5280 \mathrm{e}+1$ & $1.5863 \mathrm{e}+1$ \\
\cline { 2 - 5 } & $\mathrm{B}$ & $2.2261 \mathrm{e}+1$ & $1.6462 \mathrm{e}+1$ & $1.7217 \mathrm{e}+1$ \\
\hline \multirow{2}{*}{$\mathrm{V}_{\mathrm{T}} / \mathrm{T}_{\mathrm{I}}$} & $\mathrm{A}$ & $1.7603 \mathrm{e}+1$ & $1.0015 \mathrm{e}+1$ & $1.1600 \mathrm{e}+1$ \\
\cline { 2 - 5 } & $\mathrm{B}$ & $1.9364 \mathrm{e}+1$ & $1.0925 \mathrm{e}+1$ & $1.2728 \mathrm{e}+1$ \\
\hline \multirow{2}{*}{$\mathrm{f} / \mathrm{V}_{\mathrm{T}}$} & $\mathrm{A}$ & $1.5938 \mathrm{e}+1$ & $8.6806 \mathrm{e}+0$ & $9.9702 \mathrm{e}+0$ \\
\cline { 2 - 5 } & $\mathrm{B}$ & $1.7115 \mathrm{e}+1$ & $9.4765 \mathrm{e}+0$ & $1.0916 \mathrm{e}+1$ \\
\hline
\end{tabular}




\section{METHODOLOGY AND RESULTS}

For evaluated the wavelet transform, four statistic parameters were calculated for the coefficients of the DWT.

Arithmetic mean (M). It is the central tendency of a collection of numbers taken as the sum of the numbers divided by the size of the collection.

Standard deviation (SD). It shows how much variation or dispersion exists from the average. A low standard deviation indicates that the data points tend to be very close to the mean; high standard deviation indicates that the data points are spread out over a large range of values.

Interquartile range (IQR). It is equal to the difference between the first and third quartiles.

Kurtosis $(K)$. It is a measure of whether the data are peaked or flat relative to a normal distribution. That is, data sets with high kurtosis tend to have a distinct peak near the mean, decline rather rapidly, and have heavy tails. Data sets with low kurtosis tend to have a flat top near the mean rather than a sharp peak.

In this way, 28 new time series were obtained for each patient (Fig. 2), that are the features of the system. The time series are normalized (Eq. 5).

$$
x_{\text {norm }}=\frac{x-\min (x)}{\max (x)-\min (x)}
$$

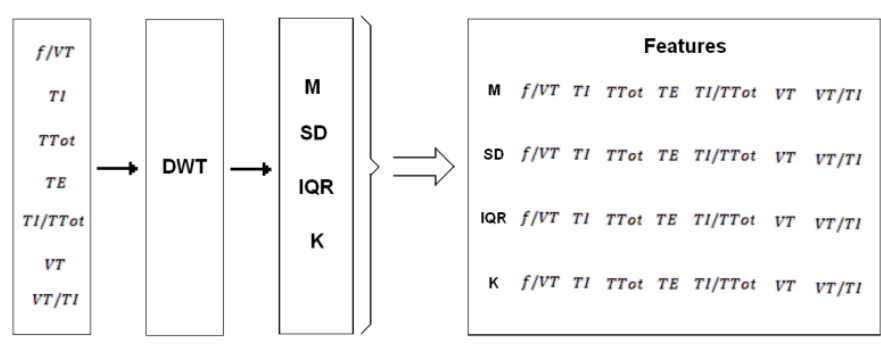

Fig2. Representation of 28 features for each patient with seven respiratory time series

Multilayer perceptrons. A multilayer perceptron (MLP) is a feedforward artificial neural network model that maps sets of input data onto a set of appropriate output. An MLP consists of multiple layers of nodes in a directed graph, with each layer fully connected to the next one. Except for the input nodes, each node is a neuron (or processing element) with a nonlinear activation function. MLP uses a supervised learning technique called backpropagation for training the network.

The network architecture selected for this investigation was of four input neurons, one hidden layer and one neuron of output. The newff command, of the Neural Network Toolbox Matlab, was used for to train the neural network. Of the 66 files of the database, 40 files were designated for the training of the neural network and 26 for the stage of validation. Since each patient is characterized with 28 series and it is desired to set four input variables for the neural network, it has 20475 possible combinations of variables. An algorithm was developed for to select among the all possible combinations and for each group of variables was modified the number of neurons in the hidden layer, of one to eleven. The figure 3 shows the change of MSE index of the stage of validation in function of the neurons of the hidden layer and the combination. The best combination of variables was obtained for $\mathrm{MVT}_{\mathrm{T}}, \mathrm{SDV}_{\mathrm{T}}, \mathrm{KT}_{\mathrm{T}}$ and $\mathrm{IQRT}_{\mathrm{T}}$ ot with four neurons in the hidden layer. The MSE was of 0.077. Table III presents the results of validation stage.

TABLE III

RESULTS OF THE VALIDATION STAGE - MLP

\begin{tabular}{|c|c|c|}
\cline { 2 - 3 } \multicolumn{1}{c|}{} & Group A & Group B \\
\hline Group A (13 Patients) & 11 & 2 \\
\hline Group B (13 Patients) & 0 & 13 \\
\hline
\end{tabular}

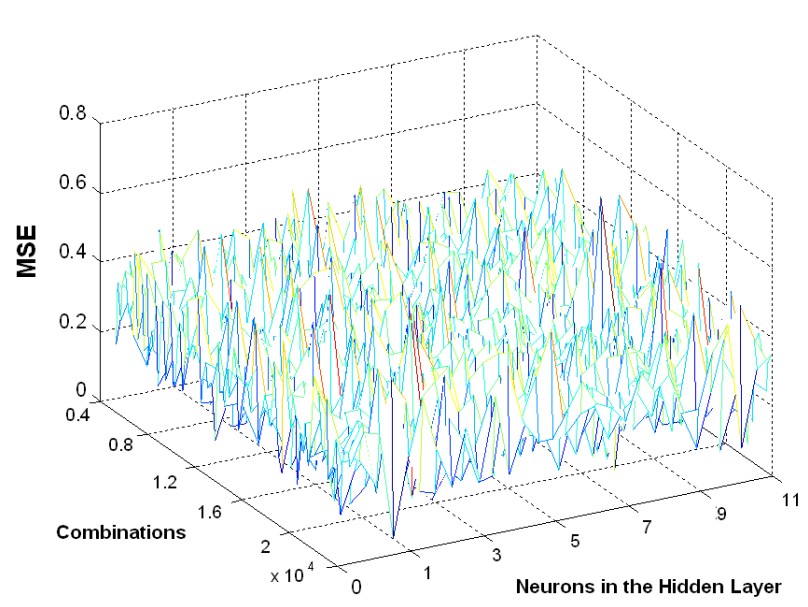

Fig. 3 Neurons in the Hidden Layer vs. Combinations

\section{CONCLUSIONS}

A methodology based on DWT and neural networks has been applied for determine the moment of disconnection of patients of the mechanical ventilation, analyzing the respiratory pattern. This diagnosis is a critical task for medical experts in hospital environments. Most decisions in this context bound to be made on the basis of doctors' experience.

\section{REFERENCES}

[1] N. Patroniti. Mechanical ventilation: skills and techniques. European society of intensive care medicine, 2011.

[2] M. Meade, G. Guyatt, D. Cook, L. Griffith, Predicting success in weaning from mechanical ventilation, Chest, 120, 6, 2001.

[3] M.J. Tobin, Advances in mechanical ventilation, N. Engl. J. Med., Vol. 344, N. 26, pp. 1986-1996, 2001.

[4] G. Benchetrit, Breathing pattern in humans: diversity and individuality, Respiration Physiology, 122, pp. 123-129, 2000.

[5] P. Caminal, L. Domingo, B.F. Giraldo, M. Vallverdú, S. Benito, G.Vázquez, D. Kaplan, Variability analysis of the respiratory volume based on nonlinear prediction methods, Medical \& Biological Engineering \& Computing, 42, pp. 86-91, 2004. 
[6] M.J. Tobin, M.J. Mador, S.M. Guenter, R.F. Lodato, M.A. Sackner, Variability of resting respiratory center drive and timing in healthy subjects, J. Appl. Physiol., 65, pp. 309-317, 1988.

[7] E.N. Bruce, Measures of respiratory pattern variability, in Bioengineering approaches to pulmonary physiology and medicine, Plenum Press, pp. 149-160, 1996.

[8] M.C. Khoo, Determinants of ventilatory instability and variability, Respiratory Physiology, 122, pp. 167-182, 2000.

[9] B. Giraldo, C. Arizmendi, E. Romero, R. Alquezar, P. Caminal, S. Benito, D. Ballesteros, Patients on weaning trials from mechanical ventilation classified with neural networks and feature selection. Proceedings of the $28^{\text {th }}$ IEEE EMBS Annual International Conference New York City, USA, 2006

[10] B. Giraldo, A. Garde, C. Arizmendi, R. Jané, S. Benito, I. Díaz, D. Ballesteros, Support vector machine classification applied on weaning trials patients. Proceedings of the $28^{\text {th }}$ IEEE EMBS Annual International Conference New York City, USA, 2006

[11] C.A. Arizmendi, E. Romero, R. Alquezar, P. Caminal, I. Díaz, S. Benito, B.F. Giraldo, Data mining of patients on weaning trials from mechanical ventilation using cluster analysis and neural networks. Engineering in Medicine and Biology Society, 2009. EMBC 2009. Annual International Conference of the IEEE.

[12] Mallat, S. (1999). A wavelet tour of signal processing. San Diego (CA), USA: Academic Press. 